FM 6:

X-ray Surveys of the Hot and Energetic Cosmos 


\title{
X-ray Surveys of the Hot and Energetic Cosmos
}

\author{
Andrea Comastri ${ }^{1}$ and C. Megan Urry ${ }^{2,3}$ \\ ${ }^{1}$ INAF-Osservatorio Astronomico di Bologna, Via Ranzani 1 40127, Bologna, Italy \\ email: andrea.comastri@oabo.inaf.it \\ ${ }^{2}$ Department of Physics, Yale University, P.O. Box 208121, New Haven, CT 06520, USA, \\ ${ }^{3}$ Yale Center for Astronomy \& Astrophysics, Physics Department, P.O. Box 208120, New \\ Haven, CT 06520, USA,
}

\begin{abstract}
A science meeting is an opportunity to exchange ideas with colleagues, to hear of new results and to learn from comprehensive reviews of a topic. Much of it happens in the meeting room and much of it also happens in the corridors of the meeting venue and in restaurants and perhaps bars near the meeting location. Its a combination of people and of place that is a bit [hard to predict] but when it goes well, you know it.

All these elements came together for IAU Focus Meeting 6, X-ray Surveys of the Hot and Energetic Cosmos, in Honolulu last August. There are not many places more pleasant for an astronomical meeting than Hawaii, and the speakers did an outstanding job of reviewing the field and relaying the latest results.

X-ray surveys have been a staple of astrophysics for nearly 50 years. There are large surveys and small, deep surveys and shallow, soft X-ray energies and hard. The combination gives us invaluable information about the hottest and/or most relativistic environments known. Theory helps us interpret the data in terms of the underlying physics. The heady combination of all of the above shaken and mixed in Hawaiian paradise has given us all a deeper understanding of the Universe. Please read on to see why.
\end{abstract}

\section{X-ray surveys}

$\mathrm{X}$-ray surveys are playing a key role in our understanding of the hot and energetic component of our Universe. Since their launch, in 1999, both XMM-Newton and Chandra surveys have transformed our understanding of the sources of the Cosmic $\mathrm{X}$-ray Background, including their cosmological evolution and the physical processes shaping their properties. Detailed investigations of the physics and evolution of $\mathrm{X}$-ray selected sources were addressed thanks to massive observations along the entire electromagnetic spectrum (COSMOS, ECDFS, AEGIS, etc). The X-ray survey science is still actively pursued by the scientific community and well received by XMM and Chandra Time Allocation Committee who have recently granted major programs (i.e. the Chandra Ultradeep 7Ms Survey in the CDFS, the 3Ms COSMOS Legacy Survey and the Large area surveys with XMM: XXL and Stripe82X).

The launch of NuSTAR in 2012, with its imaging capabilities at $>8 \mathrm{keV}$, is providing a unique opportunity to map the third dimension of the luminosity-redshift-obscuration space at limiting fluxes more than two orders of magnitude fainter than those reached by non imaging Swift/BAT and INTEGRAL surveys. The key goal of NuSTAR surveys is to resolve a significant fraction of the hard $\mathrm{X}$-ray background close to its peak in the energy density at 25-30 keV, and elucidate whether it is due to the most heavily obscured Compton-thick AGN. The Japanese mission ASTRO-H, due for launch in 2016, will also survey the hard X-ray sky above $10 \mathrm{keV}$. The broad band coupled with the highest energy 


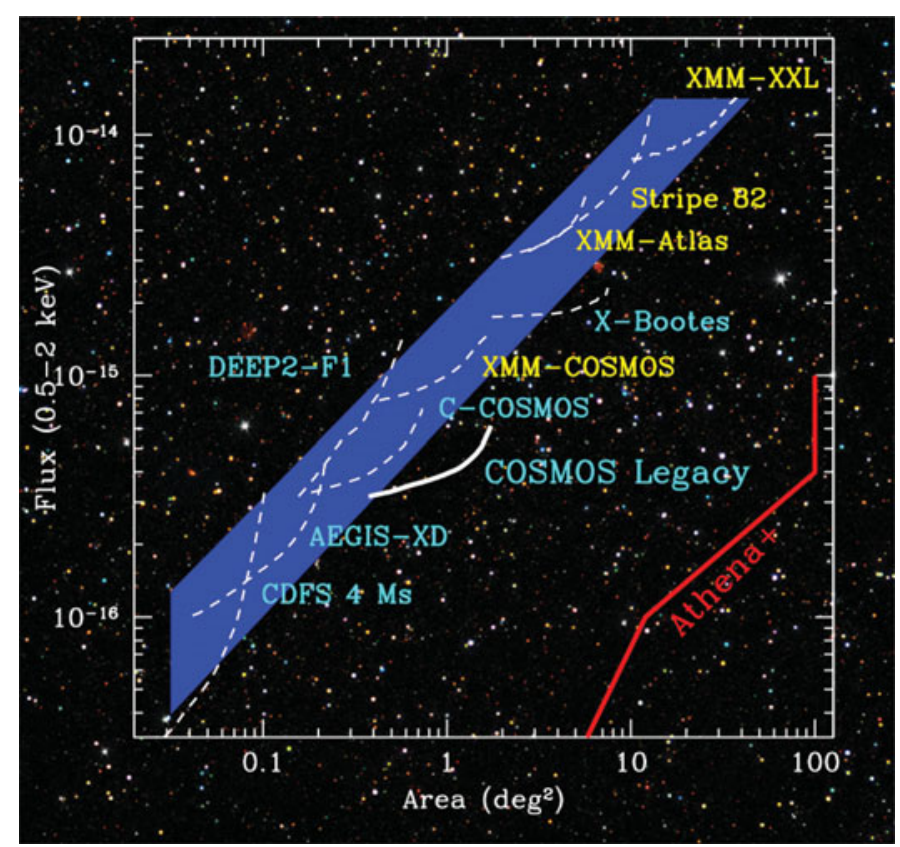

Figure 1. Area-flux curves for contiguous Chandra (cyan) and XMM (yellow) contiguous X-ray surveys. Each survey has been plotted using each sensitivity curve starting from the flux corresponding to the area that is $80 \%$ of the maximum area for that survey to the flux corresponding to the $20 \%$ of the total area. The survey locus (blue shaded area) drawn by our current X-ray telescopes is shown: COSMOS Legacy is exploring a new region off the survey locus by using a total exposure time which is unusual (4.8 Ms total) for that given area flux combination, moving towards the region occupied by future mission surveys like Athena (red curve). The background is the 3 color X-ray mosaic of the C-COSMOS survey.

resolution ever achieved in the $3-10 \mathrm{keV}$ band will open an enormous discovery space for a wide variety of objects and especially accreting black holes and cluster of galaxies. eROSITA, will survey the entire sky starting in 2017. In the soft, $0.5-2 \mathrm{keV}$ band, it will be about 20 times more sensitive than the ROSAT all sky survey, while in the hard band $(2-10 \mathrm{keV})$ it will provide the first ever imaging survey of the sky at those energies. Such a sensitive all-sky survey will revolutionize our view of the high-energy sky. The detection of very large samples $\left(\sim 10^{5}\right.$ objects) of galaxy clusters out to redshifts $z>1$, in order to study the large scale structure in the Universe, test and characterize cosmological models including Dark Energy will provide a major breakthrough. eROSITA is also expected to yield a sample of around 3 millions Active Galactic Nuclei, providing a unique view of the evolution of supermassive black holes within the emerging cosmic structure.

The title of the IAU Focus Meeting number 6 "X-ray surveys of the hot and the energetic Cosmos" is also the science theme of the ESA Large mission ATHENA foreseen for a 2028 launch. The mission aims to explore how ordinary matter assembles into the large scale structure and how black holes grow. Thanks to the high spectral resolution Integral Field Unit, and the Wide Field Imager at the focus of a 2 square meters effective area X-ray telescope, ATHENA will guarantee major breakthroughs and provide transformational science.

The aim of the FM6 was to review and discuss the recent observational advances obtained by X-ray surveys complemented by multi-wavelength follow-up programs, alongside progress in theory/simulation. The scientific program, distributed over 7 sessions, 
touched most of the "hot and energetic" questions about the physics and the cosmology of cluster of galaxies, the light up and cosmic evolution of accreting black holes and the high energy phenomena in starbursts and normal galaxies. The final round table, chaired by Guenther Hasinger IfA director, and with the presence of top level researchers in the field, addressed the way forward of future missions including synergies with multiwavelength facilities. 